\title{
Variações no comprimento dos indivíduos de uma população do mexilhão dourado, Limnoperna fortunei (Mollusca: Bivalvia: Mytilidae), ao longo do ano, na Praia do Veludo, Lago Guaíba, Rio Grande do Sul, Brasil
}

\author{
Cintia P. dos Santos ${ }^{1}$; Maria Cristina D. Mansur ${ }^{1} \&$ Norma L. Würdig ${ }^{2}$ \\ ${ }^{1}$ Museu de Ciências e Tecnologia, Pontifícia Universidade Católica do Rio Grande do Sul. Avenida Ipiranga 6681, \\ 90619-900 Porto Alegre, Rio Grande do Sul, Brasil. E-mail: cipinheiro@yahoo.com.br; mcmansur@terra.com.br \\ ${ }^{2}$ Laboratório de Invertebrados Bentônicos, Departamento de Zoologia, Universidade Federal do Rio Grande do Sul. Avenida \\ Bento Gonçalves 9500, 91501-970 Porto Alegre, Rio Grande do Sul, Brasil. E-mail: wurdignl@ufrgs.br
}

\begin{abstract}
Length variation over a one-year period in specimens of the golden mussel, Limnoperna fortunei (Mollusca: Bivalvia: Mytilidae) from Veludo Beach, Guaíba Lake, Rio Grande do Sul, Brazil. The individual size of Limnoperna fortunei (Dunker, 1857) was evaluated twice a month, for a year, based on samples from Veludo Beach $\left(30^{\circ} 12^{\prime} 35^{\prime \prime}\right.$ S, 51 $\left.11^{\prime} 68^{\prime \prime} \mathrm{W}\right)$ in Guaiba Lake, southern Porto Alegre, Brazil. Physical and chemical data on water quality, such as temperature, transparency, depth, $\mathrm{pH}$, dissolved oxygen, phosphorus, nitrogen, chlorophyll "a", and conductivity were also recorded. Adult samples were collected from underwater "sarandi" branches, Cephalanthus glabratus (Spreng) K. Schum (Rubiaceae), an aquatic macrophyte available at each site. Adults placed on artificial substrates were also checked every six months. Data were analyzed by applying multivariate statistics techniques related to the physical and chemical variables of the water and the density of individuals to estimate the patterns of the individual growth over the year. The adults were separated into three groups according to length classes: $\mathrm{Gl}, \mathrm{G} 2$ and $\mathrm{G} 3$ (groups 1 through 3). These three groups are related to the differences in behavior, namely, the ability of locomotion and fixation capacity. Individuals from Group 1 (5 to $7 \mathrm{~mm}$ in length) were the most abundant and were present in all the sampled months. Adults from artificial substrates were shown to display significant differences in seasonal growth and faster growth during spring and summer months. KEY WORDS. Invasior; length rate; seasonal growth.
\end{abstract}

RESUMO. O crescimento individual de Limnoperna fortunei (Dunker, 1857) foi avaliado com base em amostragens quinzenais realizadas na Praia do Veludo (30`12'35"S, 51 ${ }^{\circ} 11^{\prime} 68^{\prime \prime} W$ ), Lago Guaíba, ao sul do município de Porto Alegre, ao longo de um ano. Análises físico-químicas da água avaliadas foram: temperatura, transparência, profundidade, $\mathrm{pH}, \mathrm{OD}$, fósforo, nitrogênio, clorofila "a" e condutividade. As amostras de indivíduos adultos foram recolhidas junto aos ramos submersos de "sarandi", Cephalanthus glabratus (Spreng) K. Schum (Rubiaceae), disponíveis no local. Paralelamente, foram colocados substratos artificiais de cerâmica para avaliação dos indivíduos adultos assentados a cada semestre e ao final do ano. Os dados foram analisados empregando-se técnicas estatísticas multivariadas relacionando-se às variáveis físicas e químicas da água às densidades dos indivíduos. Objetivando estimar os padrões de crescimento dos indivíduos ao longo do ano observou-se que as análises de ordenação e agrupamento separaram a população em três grupos conforme as classes de comprimento: G1, G2 e G3 (grupos 1 a 3). Estes três grupos estão também relacionados às diferenças de comportamentos quanto à habilidade de locomoção e fixação. Constatou-se a predominância do grupo 1 ( 5 a $7 \mathrm{~mm}$ de comprimento), em todos os meses amostrados. Os indivíduos avaliados sobre substrato artificial demonstraram diferença significativa no crescimento sazonal, onde os indivíduos cresceram mais nos meses de primavera e verão.

PALAVRAS-CHAVE. Classes de tamanho; crescimento sazonal; invasor.

O bivalve invasor de origem asiática conhecido como mexilhão dourado, Limnoperna fortunei (DUNKER 1857), foi registrado pela primeira vez no Brasil no ano de 1998, nos arredores de Porto Alegre (Mansur 1999, 2003), no extremo sul do Brasil e nas proximidades de Corumbá no centro-oeste do país. Estas populações, segundo MANsur et al. (2004) seriam oriundas de uma invasão anterior desta espécie que ocorreu na América do Sul, registrada para a Argentina, em torno de 1991 (PAstorino et al. 1993, DARRIGRAN \& PASTORINo 1995, 2003). No primeiro caso, a introdução teria ocorrido através da água de lastro de navios de 
cabotagem vindos de portos dulcícolas do baixo rio Paraná e rio da Prata, que entrando na laguna dos Patos pelo canal de Rio Grande, alcançaram os portos do lago Guaíba. No segundo, por migração passiva a montante dos rios Paraná e Paraguai, auxiliada pela navegação interior (MAnsur et al. 2004). Segundo DarRigran (2002) o mexilhão dourado, devido ao seu grande poder reprodutivo, crescimento rápido e comportamento gregário, forma grandes aglomerados em curto espaço de tempo causando problemas de obstrução nas canalizações e entradas de água para o abastecimento das cidades.

Durante os dez anos de invasão do mexilhão dourado na Argentina, DARRIGRAN (2002) observou um aumento gradativo quanto à densidade populacional do mexilhão dourado. No início encontraram agrupamentos de quatro a cinco ind $/ \mathrm{m}^{2}$ (PAstorino et al. 1993). Após quatro anos, constataram um pico de densidade em torno de $100000 \mathrm{ind} / \mathrm{m}^{2}$ seguido de um decréscimo para $40000 \mathrm{ind} / \mathrm{m}^{2}$, que se manteve. Mansur et al. (2003), com base em amostragens quantitativas, constataram no Guaíba, em 1998 e 1999, pequenos aglomerados formados por poucos indivíduos, em dois anos a população chegou a $140000 \mathrm{ind} / \mathrm{m}^{2}$ e depois estabilizou em médias de 40000 a 80000 ind $/ \mathrm{m}^{2}$ nos aglomerados.

A dinâmica populacional da espécie foi pesquisada por MorTon (1977) num reservatório de água para o abastecimento da cidade de Hong Kong. Através de amostragens mensais, o autor observou o crescimento do molusco e os períodos de assentamento das larvas, que ocorreram três vezes ao ano, com períodos de duração distinta e que foram relacionados às alterações de temperatura e de oxigênio dissolvido na água.

Morton (1982) revelou os primeiros dados sobre a reprodução de L. fortunei de populações oriundas de Hong Kong. Constatou que a espécie é essencialmente dióica (sem a ocorrência de hermafroditas) com desova bianual influenciada pelas variações sazonais da temperatura da água. DARRIGRAN et al. (1998) mencionaram a presença de hermafroditas (0,55\%) em amostras da população de L fortunei da Argentina. DarRIgran et al. (1999) concluíram que, nos primeiros anos de invasão, a reprodução fora praticamente contínua, sem uma sincronização nos padrões de reprodução. No terceiro ano, foi possível reconhecer dois picos anuais de desova, semelhante ao comportamento reprodutivo da população asiática.

IWASAKI \& URYu (1998), após estudos de uma população de L. fortunei do Japão, constataram que a reprodução ocorre uma vez ao ano. O recrutamento foi observado no período de verão. Quanto ao crescimento populacional para os indivíduos adultos observaram uma estrutura com tendência bimodal. Distinguiram duas coortes conforme o tamanho, porém em certos meses do ano, estas se confundem em uma só. A longevidade estimada para esta população seria no máximo de dois anos, não havendo sobreviventes no terceiro ano.

Magara et al. (2001) estudaram outras populações do mexilhão dourado no Japão, observando que longevidade e a capacidade reprodutiva do $L$. fortunei não ultrapassaram o se- gundo ano. A reprodução foi anual, mas se estendeu por um período mais longo (maio a setembro) conforme observado por Iwasaki \& Uryu (1998). Por sua vez, Goto (2002) descreveu que, na localidade de Kobe, também no Japão, L. fortunei liberou gametas no verão, cresceu $20 \mathrm{~mm}$ até o final do primeiro ano, quando ocorreu a mortalidade da população.

Constatadas diferenças nas características reprodutivas e comportamentais, do L. fortunei, dependentes de variações climáticas, latitudinais e também do período de invasão, o presente estudo teve por objetivo caracterizar o crescimento anual dos indivíduos de L. fortunei na Praia do Veludo, lago Guaíba, através das medidas de comprimento dos indivíduos adultos aglomerados sobre vegetação ciliar nativa e sobre substrato artificial.

\section{MATERIAL E MÉTODOS}

A área de estudos compreende a Praia do Veludo junto ao trapiche da Associação dos Pescadores (APESCA) (30¹2'35"S, $51^{\circ} 11^{\prime} 68^{\prime \prime} \mathrm{W}$ ), na margem esquerda do lago Guaíba, Bairro Belém Novo, ao sul do município de Porto Alegre, onde predonima o clima subtropical (Fig. 1). Foram realizadas coletas quinzenais acompanhadas de amostragens de larvas do plâncton e da água, no período da manhã, de setembro de 2002 a dezembro de 2003. As larvas foram coletadas com rede de plâncton com 30 $\mu \mathrm{m}$. Foram medidas, a temperatura da água e do ar, a profundidade e a transparência, junto a um pilar demarcado no trapiche. As amostras de água, para análise de oxigênio dissolvido, condutividade, $\mathrm{pH}$, nitrogênio total, fósforo total, sólidos em suspensão (АвNт 1986, 1989 a,b, 1992, 1997, 1999) e clorofila "a" (Сетеsв 1987), foram recolhidas em frascos esterilizados e mantidos sob refrigeração, até seu processamento no Laboratório de Processos Ambientais (LAPA-PUCRS).

A coleta de indivíduos adultos de $L$. fortunei efetivou-se junto às partes submersas do "sarandi", vegetação ripária da macrófita Rubiaceae, Cephhalanthus glabratus (Spreng.) K. Schum. Foram selecionados aleatoriamente ramos que tivessem aproximadamente três centímetros de diâmetro e que fossem cobertos por aglomerados de mexilhão. Cada amostra foi acondicionada em saco plástico, levada ao laboratório, onde foi seccionada para obtenção de três réplicas padronizadas de aproximadamente $10 \mathrm{~cm}^{2}$, conservadas em álcool $70 \%$. Os mexilhões foram destacados, contados e medidos quanto ao seu comprimento máximo, utilizando-se um paquímetro digital de 0,01 mm de precisão (Mitutoyo). Considerou-se o comprimento máximo como a distância da extremidade anterior, situada logo abaixo e à frente dos umbos, até a extremidade posterior da concha. As medidas de comprimento foram reunidas conforme intervalos de $2 \mathrm{~mm}$ (5-7, 7-9,.. 36-38), totalizando 17 classes de comprimento dividido em 16 intervalos conforme VAzzoler (1981). Considerou-se indivíduos adultos a partir de cinco milímetros de comprimento conforme DARRIGRAN et al. (1999), servindo de base para a definição da classe mínima de tamanho da população adulta. Indivíduos abaixo destas medi- 


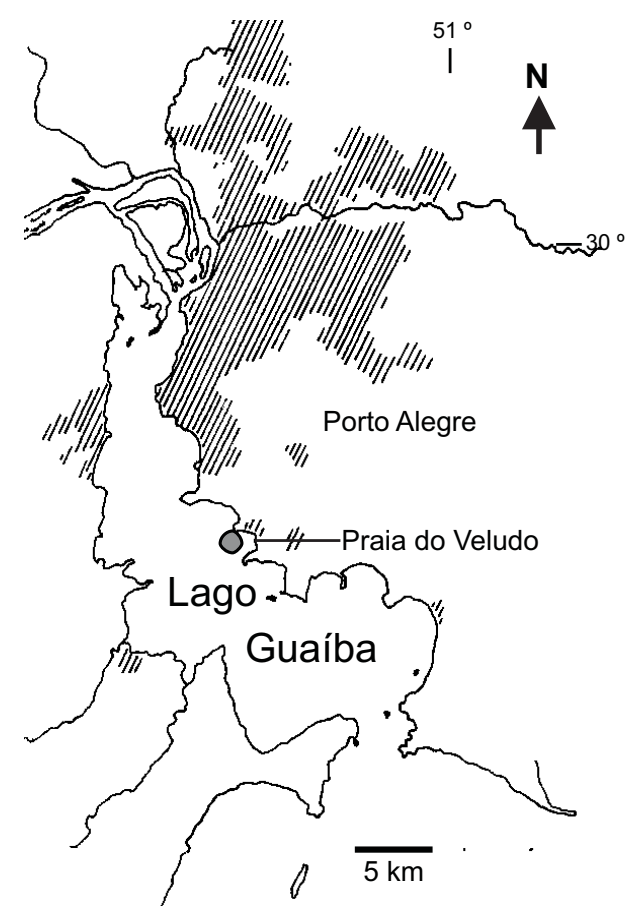

Figura 1. Mapa indicando o local de amostragem: Praia do Veludo, Belém Novo, margem esquerda do lago Guaíba, Porto Alegre, Rio Grande do Sul.

das, logo após o momento em que deixam de ser larvas planctônicas, foram considerados recrutas e não foram incluídas nos intervalos selecionados. Além da separação nas classes acima, foi realizada a contagem absoluta dos adultos.

Para acompanhamento do crescimento utilizou-se substrato artificial, tijolos vazados de cerâmica (Figs 2-3), com seis superfícies retangulares. Apenas as quatro superfícies maiores medindo aproximadamente $14 \times 10 \mathrm{~cm}$, foram avaliadas, sendo duas vazadas e duas caneladas. As duas menores não foram consideradas devido a dificuldade de observação junto à lupa). A estrutura vazada compreende seis perfurações de $3 \mathrm{~cm}$ e duas de $1 \mathrm{~cm}$ de diâmetro. Foram montados seis conjuntos de quatro tijolos cada, sustentados por suportes de ferro (Figs 2-3). Estes tijolos, permaneceram suspensos entre os juncais a uma altura de $20 \mathrm{~cm}$ do fundo. Foram substituídos a cada seis meses e retirados ao final de um ano. Sob influência de fortes tempestades ocorridas no mês de outubro de 2002, três suportes foram arrastados e parcialmente cobertos pelo substrato. Devido a esta perda parcial, apenas os três restantes, foram utilizados para avaliação. Na contagem e medida do comprimento máximo dos indivíduos adultos coletados nestes tijolos, foram consideradas somente as quatro superfícies maiores, ou seja, os dois lados retangulares com superfície canelada e dois lados contendo seis perfurações. Cada perfuração foi observada até a profundidade de 1 cm (Figs 2-3). Esta área também foi computada no cálculo da superfície amostrada, somando ao todo $463 \mathrm{~cm}^{2}$ para cada tijolo. Os recrutas não foram mensurados, mas quantificados em termos de densidade e incluídos no trabalho.

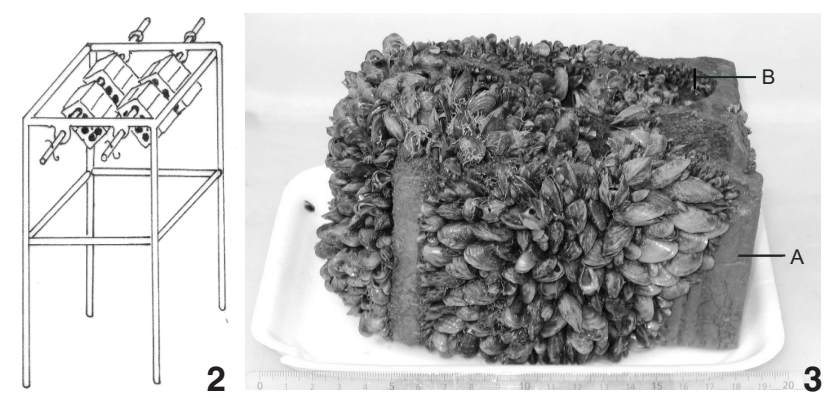

Figuras 2-3. Substrato artificial para acompanhamento do crescimento dos indivíduos adultos, na Praia do Veludo: (2) Desenho esquemático do suporte de ferro; (3) tijolo: (A) superfície avaliada, (B) superfície e perfurações avaliadas até a profundidade de $1 \mathrm{~cm}$.

As variáveis físicas e químicas foram contrapostas às densidades de indivíduos adultos coletados nos sarandis para estimar relações com o crescimento da população ao longo do ano. Empregou-se a análise exploratória baseada em técnicas multivariadas de correlação, ordenação, agrupamento e congruência, utilizando-se os softwares MULTIV e SYNCSA (Pillar 2001, 2002, 2004).

Com objetivo de testar a estabilidade dos eixos de ordenação e a nitidez dos grupos gerados, foram realizados testes de auto-reamostragem bootstrap, que além da confiabilidade dos resultados, fornece informações sobre a suficiência amostral (Pillar 1998, 1999a, b). O limiar de probabilidade utilizado para a interpretação dos resultados foi p = 0,05 (Pillar 1999a, b).

A medida de semelhança utilizada foi à distância de corda entre unidades amostrais, com normalização e centralização dos dados implícita. Esta medida de semelhança é utilizada em dados onde às ausências são bastante comuns (OrLóci 1967). Os agrupamentos hierárquicos de unidades amostrais foram realizados pelo método de variância mínima, ou soma de quadrados (WARD 1963, Orlóci 1967). A análise de ordenação de unidades amostrais foi realizada pelo método de coordenadas principais (PCoA) (Gower 1966).

Além das análises descritas, para avaliar as máximas correlações individualmente nos grupos formados pelas classes de comprimento, utilizou-se a correlação de Pearson $\mathrm{p}<0,05$.

Para a avaliação do crescimento sazonal dos adultos, avaliaram-se os tijolos semestrais (controle do experimento), agrupando as estações do ano primavera-verão e outono-inverno, sendo que cada tijolo foi considerado como uma réplica. As estações foram comparadas através do teste estatístico MannWhitnney $\left(\mathrm{H}_{0}: \mu \mathrm{PV}=\mu \mathrm{OI}\right)$, com nível de significância de $95 \%$. Ambos os testes foram utilizados com auxílio do software SPSS 11.5 . 


\section{RESULTADOS}

A distribuição sazonal dos valores referentes aos indicadores físicos e químicos da estação de amostragem na Praia do Veludo é apresentada na tabela I.

Tabela I. Média das variáveis físicas e químicas de dezembro 2002 a dezembro de 2003.

\begin{tabular}{lrrrrr}
\hline \multicolumn{1}{c}{ Variáveis } & Média & $\begin{array}{c}\text { Erro } \\
\text { padrão }\end{array}$ & $\begin{array}{r}\text { Desvio } \\
\text { padrão }\end{array}$ & Mín. & Máx. \\
\hline Água $\left({ }^{\circ} \mathrm{C}\right)$ & 20,79 & 0,95 & 4,75 & 12,00 & 27,50 \\
Condutividade $(\mathrm{mS} / \mathrm{cm})$ & 0,06 & 0,00 & 0,02 & 0,03 & 0,09 \\
$\mathrm{pH}$ & 7,02 & 0,07 & 0,33 & 6,22 & 7,54 \\
$\mathrm{OD}(\mathrm{mg} / \mathrm{l})$ & 6,53 & 0,37 & 1,85 & 3,91 & 11,33 \\
Sólidos suspensos $(\mathrm{mg} / \mathrm{l})$ & 31,38 & 11,94 & 59,68 & 0,10 & 214,00 \\
Clorofila a $(\mu \mathrm{m} / \mathrm{l})$ & 4,46 & 1,23 & 6,14 & 0,01 & 23,90 \\
Fósforo Total $(\mathrm{mg} / \mathrm{l})$ & 0,13 & 0,02 & 0,09 & 0,01 & 0,38 \\
Nitrogênio total $(\mathrm{mg} / \mathrm{l})$ & 0,14 & 0,06 & 0,31 & 0,01 & 1,44 \\
Transparência $(\mathrm{cm})$ & 38,32 & 3,93 & 19,64 & 8,00 & 100,00 \\
Profundidade $(\mathrm{cm})$ & 77,24 & 6,25 & 31,24 & 20,00 & 154,00 \\
\hline
\end{tabular}

A quantidade de larvas (Fig. 4) variou de zero a 23 larvas por litro. Os picos de maior densidade foram observados nos meses de outubro e dezembro de 2002 e outubro de 2003 (respectivamente 23 larvas/litro, 20 larvas/litro e 17 larvas/litro). O período de menor incidência de larvas ocorreu nos meses de abril a setembro de 2003 .

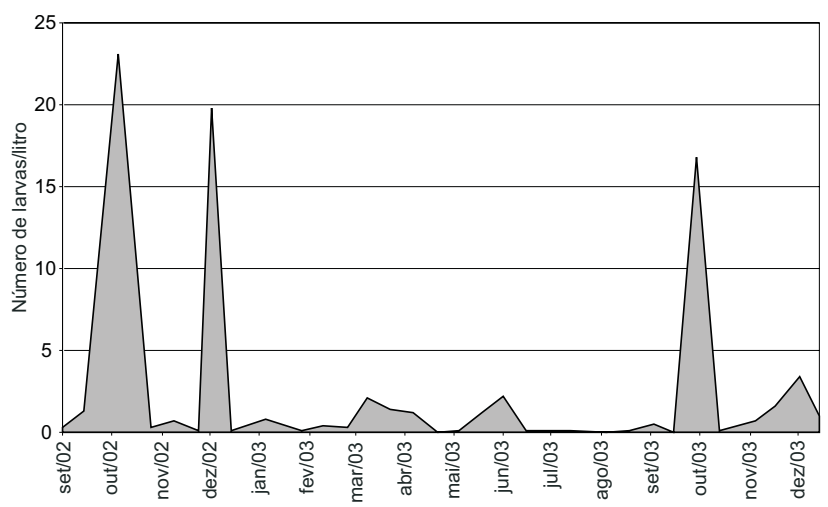

Figura 4. Número de recrutas em substrato artificial $\left(463 \mathrm{~cm}^{2}\right), L$. fortunei, durante o período de amostragem de setembro de 2002 a dezembro de 2003, na Praia do Veludo.

A quantidade média de recrutas (Fig. 5) na superfície dos substratos artificiais variou de um em setembro de 2002 a 7545 indivíduos no mês de novembro de 2003. A maior densidade

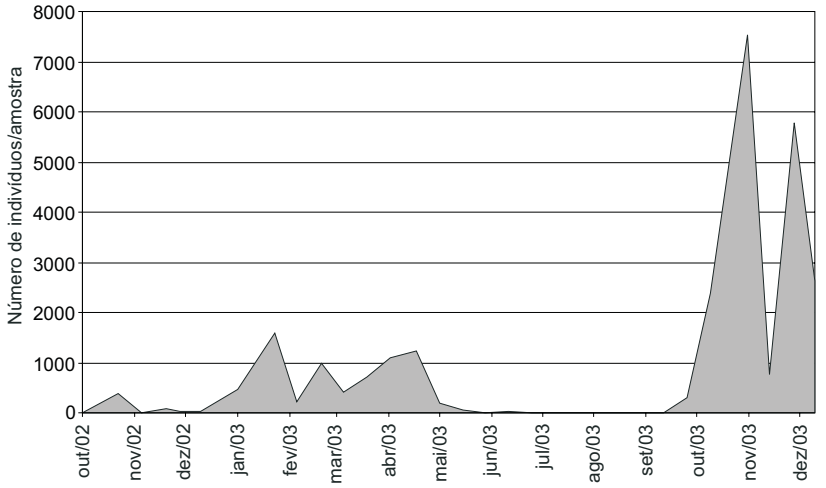

Figura 5. Presença de larvas de $L$. fortunei durante o período de amostragem de setembro de 2002 a dezembro de 2003 na Praia do Veludo.

de recrutas observou-se no período de novembro a dezembro de 2003 (respectivamente 7545 e 5771).

A quantidade total do número de adultos agregados sobre ramos de "sarandi" variou de 157 a 997 indivíduos por amostra, com média de 490 indivíduos por amostra. As maiores densidades por amostra ocorreram em fevereiro (com 997 indivíduos), março (694 indivíduos) e abril de 2003 (818 indivíduos). A menor freqüência de adultos foi no período de outubro de 2002 (157 indivíduos). A densidade populacional de adultos agregados calculada variou de $15700 \mathrm{ind} / \mathrm{m}^{2} \mathrm{em}$ outubro de 2002 a 99700 ind $/ \mathrm{m}^{2}$ em fevereiro de 2003, com média de $49032 \mathrm{ind} / \mathrm{m}^{2}$.

O comprimento médio dos indivíduos adultos em substrato natural foi de $29 \mathrm{~mm}$ e variando de 5 a $38,1 \mathrm{~mm}$, no período amostrado. Um total de 15200 indivíduos medidos e separados por classes de comprimento, conforme cada amostragem.

As classes mais representativas, com predomínio durante todo ano, foram dos indivíduos que apresentam comprimentos de 15,01 a $23 \mathrm{~mm}$. As classes menores (de 5 a $11 \mathrm{~mm}$ ) estiveram presentes em abundância nos meses de fevereiro até agosto, com baixas de setembro até janeiro. Apenas no mês de janeiro, as classes maiores (acima de 27,01 mm) foram representativas em termos de densidade. Classes intermediárias (23,01 a $27 \mathrm{~mm})$ estiveram presentes durante todo o ano com uma densidade mais elevada em setembro 2002, fevereiro e abril de 2003.

A análise de ordenação sintetizou a variação dos comprimentos conforme as datas de coleta em dois eixos principais, os quais contêm cerca de 76,12\% da variância total (Tab. II). A estabilidade encontrada nos eixos também indicou que houve suficiência amostral. Dessa forma, os resultados obtidos na ordenação facilitaram interpretações seguras a respeito dos padrões de variação nos tamanhos dos adultos.

O diagrama de ordenação das unidades amostrais (Fig. 6) sugere com clareza a formação de três agrupamentos caracterizados basicamente pelas proporções diferenciadas de comprimento por épocas do ano. O agrupamento das unidades 
Tabela II. Resultados das análises de ordenação e agrupamento das classes de comprimento dos indivíduos adultos de L. fortunei. A informação contida nos eixos refere-se à variação total dos dados. A estabilidade dos eixos e a nitidez dos grupos são probabilidades geradas através de 1000 interações de auto-reamostragem bootstrap.

\begin{tabular}{cccccc}
\hline & \multicolumn{2}{c}{ Ordenação } & & \multicolumn{2}{c}{ Agrupamento } \\
\cline { 1 - 2 } \cline { 5 - 6 } Eixo & Informação contida nos eixos $(\%)$ & Estabilidade dos eixos $P\left(\Theta_{i}^{\circ} \geqslant \Theta_{i}^{*}\right)$ & & Número de grupos & Nitidez de grupos $P\left(G^{\circ} \leqslant G^{*}\right)$ \\
\hline 1 & 56,30 & 0,032 & 2 & 0,313 \\
2 & 19,82 & 0,014 & 3 & 0,170 \\
3 & 9,21 & 0,096 & 4 & 0,072 \\
\hline
\end{tabular}

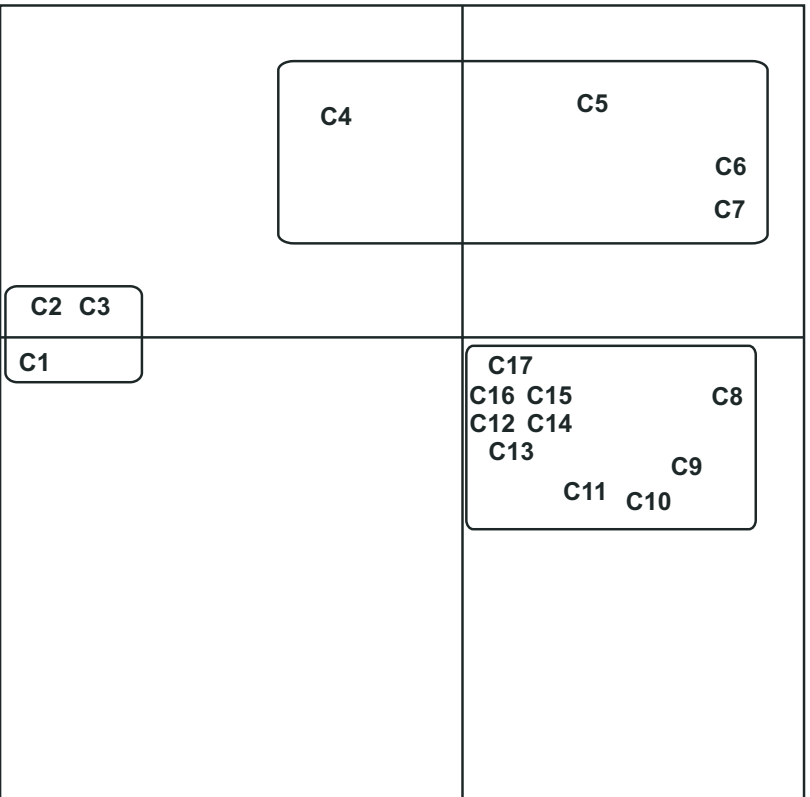

Figura 6. Diagrama de ordenação de classes de comprimento dos indivíduos adultos de L. fortunei. Foi utilizada análise de coordenadas principais com distância de corda entre unidades amostrais. Variáveis da classe de comprimento cuja correlação com os eixos $r \geqslant$ $|0,5|$ estão identificadas pelos seguintes códigos: C1(5-7 mm), C2(7$9 \mathrm{~mm}), \mathrm{C} 3(9-11 \mathrm{~mm}), \mathrm{C} 4(11-13 \mathrm{~mm}), \mathrm{C5}(13-15 \mathrm{~mm}), \mathrm{C} 6(15-17$ $\mathrm{mm}) \mathrm{C} 7(17-19 \mathrm{~mm}), \mathrm{C} 8(19-21 \mathrm{~mm}), \mathrm{C} 9(21-23 \mathrm{~mm}), \mathrm{C} 10(23-25$ $\mathrm{mm}), \mathrm{C} 11(25-27 \mathrm{~mm}), \mathrm{C} 12(27-29 \mathrm{~mm}), \mathrm{C} 13(29-31 \mathrm{~mm}), \mathrm{C} 14(31-$ $33 \mathrm{~mm}), \mathrm{C} 15(33-35 \mathrm{~mm}), \mathrm{C} 16(35-37 \mathrm{~mm}), \mathrm{C} 17(37-39 \mathrm{~mm})$. Os eixos horizontal e vertical contêm respectivamente 56,30 e 19,82\%.

amostrais resultou na formação de três grupos nítidos, $\mathrm{p}=0,170$, conforme indica o teste de auto-reamostragem bootstrap (Tab. II e Fig. 7). Os resultados dos testes de ordenação e agrupamento reuniram a população de $L$. fortunei em três grupos de acordo com o tamanho, data de coleta e dados ambientais, sendo denominadas no presente estudo respectivamente: grupo 1, grupo 2 e grupo 3.

Considerando os comprimentos máximo de cada classe de tamanho nas diferentes épocas do ano, um dos grupos (gru-

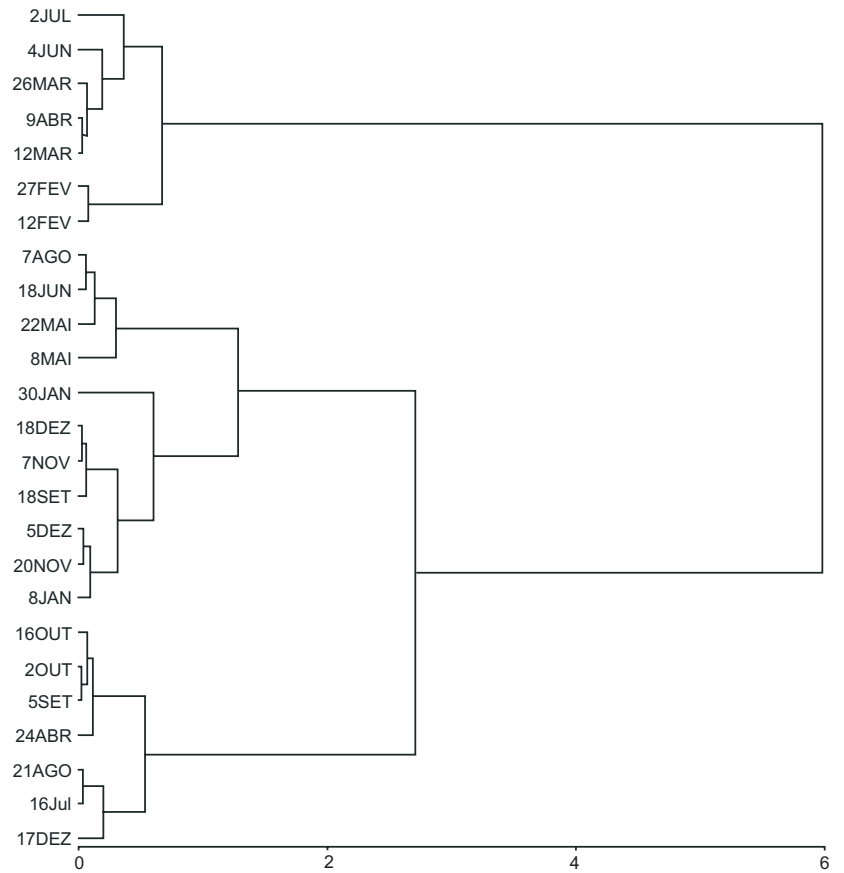

Figura 7. Dendrograma da análise de agrupamento das datas de coleta separadas pelas classes de tamanho. O agrupamento foi realizado pelo método de variância mínima com distância de corda entre unidades amostrais.

po 1) foi constituído por indivíduos que variaram de 5 a 11 $\mathrm{mm}$ (classes C1, C2 e C3). O grupo 2 foi composto por indivíduos que variaram 11 a 19 mm (classes C4, C5, C6 e C7), enquanto que o grupo 3, apresentou indivíduos com o comprimento variando entre $19 \mathrm{~mm}$ a maiores de $35 \mathrm{~mm}$ (classes $\mathrm{C} 8$, C9, C10, C11, C12, C13, C14, C15, C16 e C17).

Através do teste de correlação de Pearson, a análise das classes de tamanho reunidas não apresentou correlação significativa com as variáveis ambientais. Esta mesma análise aplicada nos grupos gerados pelo teste de agrupamento resultou: grupo 1 - não apresentou correlação significativa; grupo 2 correlação significativa com OD $(0,482)$ e sólidos suspensos $(0,5)$; grupo 3 - não apresentou correlação significativa. 
O substrato artificial (tijolos), colocado para monitoramento do crescimento da população adulta, durante o período de 12 meses apresentou indivíduos com comprimento médio de $11,63 \mathrm{~mm}$, o máximo de $29,97 \mathrm{~mm}$ e o mínimo de $2,02 \mathrm{~mm}$. Os indivíduos adultos dos amostradores semestrais apresentaram diferenças significativas no comprimento de acordo com o semestre entre as estações do ano agrupadas (teste de MannWhitney $\mu=0,05$ ) (Fig. 8). Os colocados em setembro de 2002 e retirados em março de 2003 (primavera-verão) apresentaram indivíduos com comprimento médio e erro padrão de 9,68 \pm 0,09 mm, com máximo de 20,91 e mínimo de 3,77 mm. Os colocados em março de 2003 e retirados em setembro do mesmo ano (outono-inverno) apresentaram comprimento médio e erro padrão de $6,97 \pm 0,12 \mathrm{~mm}$, o máximo de $18,05 \mathrm{~mm}$ e o mínimo de 1,65 mm. O estudo com substrato artificial permitiu observar uma nítida diferença entre as estações agrupadas (primavera-verão x outono-inverno). Existe um crescimento maior no semestre correspondente a primavera-verão, com temperatura média de $23,04^{\circ} \mathrm{C}$, máxima de $27,5^{\circ} \mathrm{C}$ e mínima de $12^{\circ} \mathrm{C}$.

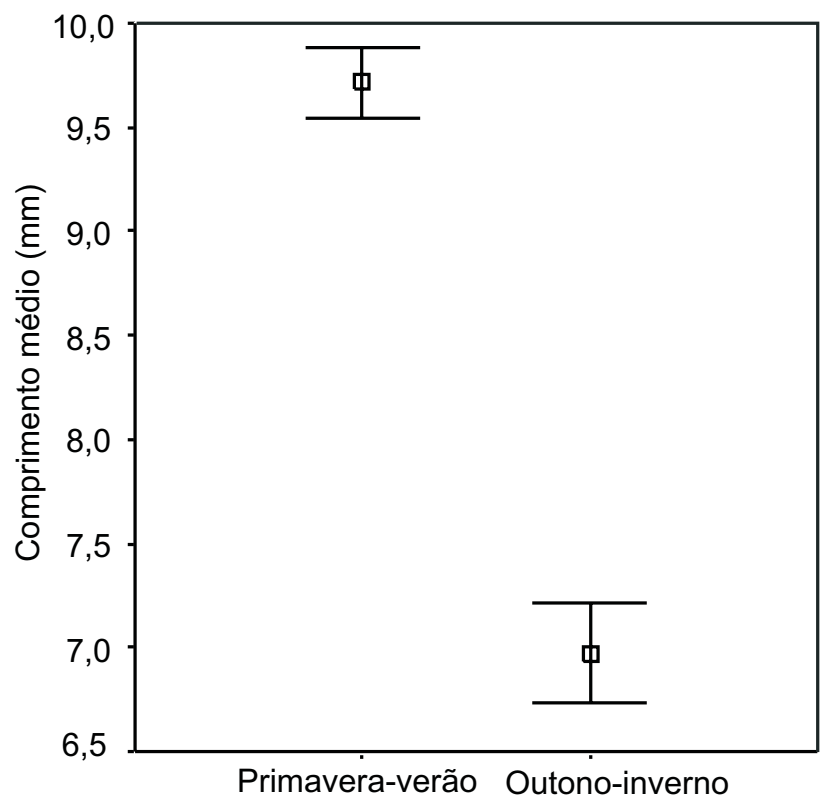

Figura 8. Gráfico da diferença no crescimento em comprimento médio nas estações do ano agrupadas. Segundo teste de MannWhitnney.

\section{DISCUSSÃO}

As espécies invasoras possuem várias características que garantem o seu sucesso no ambiente que estão invadindo. Segundo Morton (1996) e DARRIGran (2002) as características são: um curto período de vida; crescimento rápido; rápida maturidade sexual; alta fecundidade; habilidade de colonizar vários tipos de habitats; possuem uma grande tolerância fisiológica; compor- tamento gregário; muitas formas de associação com atividades humanas; vasta variabilidade genética e plasticidade fenotípica; alimentação em suspensão e possuem a habilidade de repovoar habitats previamente colonizados. O presente trabalho sobre o crescimento de L. fortunei na Praia do Veludo permitiu observar algumas características citadas anteriormente para espécies invasoras, como: comportamento gregário, altas densidades populacionais, curto período de vida e um crescimento rápido.

Comparando as medidas máximas dos indivíduos de $L$. fortunei (em substrato artificial 29,97 mm), com os comprimentos máximos observados no Japão por MaGara et al. (2001) e Gото (2002), respectivamente (1,5 e $2 \mathrm{~mm}$ ) constatou-se que os exemplares da Praia do Veludo apresentaram maior comprimento no período de um ano.

Os indivíduos de 5 a $8 \mathrm{~mm}$ (C1 e C2) encontrados em grande abundância no período de janeiro a abril de 2003, são provavelmente oriundos dos picos de recrutamento encontrados no período de outubro a dezembro de 2002 (Fig. 4). O mesmo pode-se sugerir para aos mexilhões pequenos encontrados novamente nos meses de junho a julho, como uma resposta a um pico de larvas observado em março de 2003 (Fig. 5). A presença de indivíduos adultos acima de $35 \mathrm{~mm}$, limitada ao mês de janeiro e poderia indicar que a população adulta avaliada (Grupo 3; Classe 16) alcançou seu comprimento máximo ao final do ano até o início do próximo, não sobrevivendo além dos períodos de maior calor e seca do verão. Segundo KARATAYEV et al. (2006), o mexilhão zebra (Dreissena polymorpha Pallas, 1771) morre nas temperaturas acima de $32^{\circ} \mathrm{C}$. Foi comum observar que nos períodos em que a água baixou seu nível em torno de $20 \mathrm{~cm}$ no verão de 2003, aconteceu grande mortalidade de mexilhões por ficarem, em grande parte, expostos ao calor. No entanto, no mês de janeiro de 2003 o nível da água estava em torno de $100 \mathrm{~cm}$, o que poderia ter permitido aos mexilhões um crescimento acima da média. Já no mês seguinte, frente ao calor e seca do verão, estes exemplares grandes não foram mais encontrados. A diminuição acentuada das freqüências a partir da classe de comprimento de 29 a $31 \mathrm{~mm}$, pode ser o resultado da mortalidade prevista para os indivíduos que alcançam os comprimentos maiores, observado por BAUER (1992) para outras espécies de bivalves.

Os resultados dos testes de ordenação e agrupamento reuniram a população de $L$. fortunei, em três grupos. MaroñaS (2003) também encontrou três coortes com taxas de crescimento da concha semelhantes durante o ano, no rio da Prata, Argentina. Estes grupos também estão intimamente relacionadas aos diferentes tamanhos, distintas fases de comportamento e mobilidade observados por URYu et al. (1996) ou seja, os indivíduos do grupo 1 apresentariam grande mobilidade e tigmotactismo altamente positivo (para assentamento em frestas e junto a indivíduos grandes), os indivíduos do grupo 2 com mobilidade reduzida; e os indivíduos do grupo 3 (de maior tamanho) são praticamente sem mobilidade. Estes autores constataram também que os indivíduos de comprimentos entre 5 e $12 \mathrm{~mm}$ apre- 
sentam grande mobilidade, podendo secretar e destacar o bisso até encontrar um local adequado. À medida que crescem, vão perdendo a mobilidade e acima de aproximadamente $28 \mathrm{~mm}$ não conseguem mais se deslocar no substrato. Com aproximadamente cinco milímetros de comprimento, começa o amadurecimento de suas gônadas e inicia-se a capacidade reprodutiva e o mexilhão dourado passa à fase adulta. A partir daí forma aglomerados, com vários indivíduos construindo colônias organizadas em camadas (MorTon 1977, DARRIGRAN 2002).

Cada subunidade do aglomerado é geralmente formada por uma camada de adultos maiores (grupo 3), cada um, rodeado por indivíduos de tamanhos médios (grupo 2) e os adultos jovens (grupo 1) intercalados entre todos os espaços menores formando uma superfície coesa que, aparentemente, traz vários benefícios à colônia como: defesa contra predadores e fortes correntezas. Segundo URYu et al. (1996), a presença de indivíduos grandes estimula a formação de aglomerados, pois diminui a movimentação dos mexilhões e estimula a formação do bisso pelos mais jovens.

O estudo com o substrato artificial permitiu observar uma nítida diferença no crescimento entre as estações agrupadas (primavera-verão x outono-inverno). Existe um crescimento maior no semestre correspondente a primavera-verão, com temperatura média de $23,04^{\circ} \mathrm{C}$ não ultrapassando os $30^{\circ} \mathrm{C}$. BоLтоsкoY $\&$ CATALDo (1999) citam grandes diferenças no crescimento de L. fortunei nos períodos de inverno e verão, corroborando com nossos resultados e de Karatayev et al. (2006) para mexilhão zebra (Dreissena polymorpha), onde o aumento da temperatura acelera o crescimento se esta não ultrapassar os $30^{\circ} \mathrm{C}$.

IWASAKI \& URYU (1998) afirmam a existência de variações geográficas no período reprodutivo, crescimento e longevidade do mexilhão dourado, o que justifica a grande necessidade de estudos referentes a estes temas em áreas invadidas pela espécie.

\section{AGRADECIMENTOS}

Ao CNPq pela bolsa de mestrado (Programa de Pós-graduação em Ecologia, UFRGS) concedida a CPS e de produtividade em pesquisa a MCDM (respectivamente: CNPq-132206/2002-1 e CNPq-302584/2002-1). À The Nature Conservancy do Brasil, pelo auxílio concedido - doação 018/03. A Nei de S. Antunes por ter permitido a realização dos trabalhos de campo na Sede da Associação dos Pescadores do Rio Grande do Sul e por ter auxiliado em muitas atividades durante a coleta. A Felipe R. Cardoso, Bruno M. Thormann e Paulo E. A. Bergonci pelo auxílio nas coletas e análise de dados. A Jeter J. Bertoletti, Diretor do MCT-PUCRS, pela permissão da realização de muitas atividades da pesquisa junto aos laboratórios desse Museu.

\section{LITERATURA CITADA}

AвNT. 1986. Água: determinação do pH: método do eletrométrico - NBR 9251. Rio de Janeiro, Associação Brasileira de Normas Técnicas, $6 \mathrm{p}$.
ABnT. 1989a. Água: determinação de oxigênio dissolvidométodo do eletrodo de membrana - NBR 3030. Rio de Janeiro,Associação Brasileira de Normas Técnicas, 6p.

ABNT. 1989b. Água: determinação de resíduos sólidos: método gravimétrico - NBR 10664. Rio de Janeiro, Associação Brasileira de Normas Técnicas, $14 \mathrm{p}$.

AвNT. 1992. Água: determinação de fósforo - NBR 12772. Rio de Janeiro, Associação Barasileira de Normas Técnicas, 9p.

AвNT. 1997. Água: determinação de nitrogênio orgânico, kjeldahl e total - métodos macro e semimicro kjeldahl NBR 13796. Rio de Janeiro, Associação Brasileira de Normas Técnicas, $5 \mathrm{p}$.

Aвnt. 1999. Águas minerais e de mesa: determinação da condutividade - NBR 14340. Rio de Janeiro, Associação Brasileira de Normas Técnicas, 3p.

BAUER, G. 1992. Variation in the life span of the freshwater pearl mussel. Journal of Animal Ecology 61: 425-436.

Boltovskoy, D. \& D.H. Cataldo. 1999. Population dynamics of Limnoperna fortunei, an invasive fouling mollusc, in the Lower Paraná river (Argentina). Biofouling 14 (3): 255-263.

Ceтеsв. 1987. Guia de coleta e preservação de amostras de água. São Paulo, Companhia de Tecnologia de Saneamento Ambiental, 150p.

Darrigran, G. 2002. Potential impact of filter-feeding invaders on temperate inland freshwater environments. Biological Invasions 4: 145-156.

Darrigran, G. \& G. Pastorino. 1995. The recent introduction of a freshwater asiatic bivalve Limnoperna fortunei (Mytilidae) into South America. The Veliger 32 (2): 171-175.

Darrigran, G. \& G. Pastorino. 2003. The golden mussel, Limnoperna fortunei (Dunker, 1857) (Bivalvia, Mytilidae) in the neotropical region: a 10 year story of invasion. Tentacle 11: 8-9.

Darrigran, G.; M.C. Damborenea \& P.E. Penchaszadeh. 1998. A case of hermaphroditism in the freshwater invading bivalve Limnoperna fortunei (Dunker, 1857) (Mytilidae) from Rio de la Plata, Argentina. Iberus 16 (2): 99-104.

Darrigran, G.; P.E. Penchaszadeh \& M.C. Damborenea. 1999. The reproductive cycle of Limnoperna fortunei (Dunker,1857) (Mytilidae) from a neotropical temperate locality. Journal of Shellfish Research 8 (2): 361-365.

Goто, Y. 2002. Behavior of nuisance mussel, Limnoperna fortunei, in water supply facilities. Water science and Technology 46 (11-12): 45-50.

Gower, J. C. 1966. Some distance properties of latent root and vector methods used in multivariate analysis. Biometrika 53: 325-338.

IWASAKI, K \& Y. URYu. 1998. Life Cycle of a freshwater Mytilid mussel, Limnoperna fortunei, in Uji River, Kyoto. VENUS, Japanese Journal of Malacology, 57 (2): 105-113.

Karatayev, A.I.; L.E. Burlakova \& D.K. Padilla. 2006. Growth rate and longevity of Dreissena polymorpha (PALLAS): a review and recommendations for future study. Journal of Shellfish Research 25: 23-32. 
Magara, Y; Y. Matsui; Y. Goto \& A. Yuasa. 2001. Invasion of the non-indigenous nuisance mussel, Limnoperna fortunei, into water supply facilities in Japan. Journal of Water Supply: Research and Technology AQUA 50 (3): 113-124.

Mansur, M.C.D.; L.M.Z. Richinitti \& C.P. SAntos. 1999. Limnoperna fortunei (Dunker, 1857) molusco bivalve invasor na Bacia do Guaíba, Rio Grande do Sul, Brasil. Biociências 7 (2): 147-149.

Mansur, M.C.D.; C.P. Santos; G. Darrigran; I. Heydrich; C.T. Callil \& F.R. CARdoso. 2003. Primeiro dados quali-quantitativos do mexilhão dourado, Limnoperna fortunei (Dunker), no Delta do Jacuí, no Lago Guaíba e na Laguna dos Patos, Rio Grande do Sul, Brasil e alguns aspectos de sua invasão no novo ambiente. Revista Brasileira de Zoologia 20 (1): 75-84.

Mansur, M.C.D.; C.B. Quevedo; C.P. Santos \& C.T. Callil. 2004. Prováveis vias da introdução de Limnoperna fortunei (Dunker,1857) (Mollusca, Bivalvia, Mytilidae) na bacia da laguna dos Patos, Rio Grande do Sul e novos registros de invasão no Brasil pelas bacias do Paraná e Paraguai, p. 3338. In: J.S.V. SiLVA \& R.C.C.L. Souza (Eds). Água de lastro e Bioinvasão. Rio de Janeiro, Interciências, XVIII+224p.

Maroñas, M.E.; G.A. Darrigran; E.D. Sendra \& G. Breckon. 2003. Shell growth of the goleen mussel, Limnoperna fortunei (Dunker, 1857) (Mytilidae), in the Rio de la Plata, Argentina. Hydrobiologia 1-5.

Morton, B. 1977. The population dynamics of Limnoperna fortunei (Dunker,1857) (Bivalvia: Mytilacea) in Plover Cove reservoir, Hong Kong. Malacologia 16 (1): 165-182.

Morton, B. 1982. The reproductive cycle of Limnoperna fortunei (Dunker,1857) (Bivalvia: Mytilidae) fouling Hong Kong's raw water supply system. Oceanologia et limnologia sinica 13 (4): 319-324.

Morton, B. 1996. The aquatic nuisance species: a global perspective and rewiew. In: F. D'itri (Ed.). Zebra mussels and other aquatic Species. Ann Arbor, AnnArgor Press, 54p.

OrLóCI, L. 1967. An agglomerative method for classification of plant communities. Journal of Ecology 55: 195-205.
Pastorino, G.; G. Darrigran; S. Martin \& L. Lunaschi. 1993. Limnoperna fortunei (Dunker, 1857) (Mytilidae), nuevo bivalvo invasor en aguas del río de La Plata. Neotropica 39 (101/102): 34.

Pillar, V.D. 1998. Sampling sufficiency in ecological surveys. Abstracta Botânica 22: 37-48.

PIllar, V.D. 1999a. The boostraped ordination reexamined. Journal of Vegetation Science 11: 895-902.

Pillar, V.D. 1999b. How sharp are classifications?. Ecology 80 (8): 2508-2516.

PilLAR, V.D. 2001. MULTIV: aplicativo para análise multivariada e teste de hipóteses. Porto Alegre, Departamento de Ecologia da Universidade Federal do Rio Grande do Sul, Disponível online em: http://ecoqua.ecologia.ufrgs.br/arquivos/ Software [Acessado em 30.VI.2004].

PILLAR, V.D. 2002. SYNCSA: software intergrado para análise multivariada de comunidades baseada em caracteres, dados de ambiente, avaliação e testes de hipóteses. Departamento de Ecologia da Universidade Federal do Rio Grande do Sul, Porto Alegre. Disponível online em: http:// ecoqua.ecologia.ufrgs.br/arquivos/Software [Acessado em 30.VI.2004].

Pillar, V.D. 2004. MULTIV - Multivariate Exploratory Analysis, Randomization Testing and Bootstrap Resampling. User's Guide v. 2.3. Porto Alegre, Departamento de Ecologia da Universidade Federal do Rio Grande do Sul, Available online at: http://ecoqua.ecologia.ufrgs.br [Accessed in 30.VI.2004].

URYU, Y.; K. IWASAKI \& M. HINQUE. 1996. Laboratory experiments on behaviour and movement of a freshwater mussel, Limnoperna fortunei (Dunker). Journal of Molluscan Studies 62: 327-341.

VAzzoler, A.E.A. 1981. Manual de métodos para estudos biológicos de populações de peixes. Reprodução e crescimento. Brasília, CNPq, 108p.

WARD, J.H. 1963. Hierarchical grouping to optimize an objective function. Journal of American Statistical Association 58: 236-244.

Submitted: 27.VII.2007; Accepted: 05.IX.2008.

Editorial responsibility: Georgina Bond-Buckup 